\title{
ENERGY EFFICIENT LOAD BALANCED ROUTING PROTOCOL FOR WIRELESS SENSOR NETWORKS
}

\author{
Alghanmi Ali Omar and ChongGun Kim* \\ Department of Computer Engineering, Yeungnam University, South Korea \\ alghanmia88@gmail.com, cgkimeyu.ac.kr
}

\begin{abstract}
Due to the enormous applications of wireless sensors, the research on wireless sensor networks remains active throughout the past two decades. Because of miniaturization of sensor nodes and their limited batteries, the energy efficiency and energy balancing are the demand in-need to extend the life time of sensor networks. This study proposes an energy-aware directional routing protocol for stationary wireless sensor network. The routing algorithm is non-table driven, destination aware and packet forwarder nodes are selected on the basis of admissible heuristic logical distance, and packet forwarding direction is also determined in very simplistic method. The algorithm is designed for 1-hop, 2-hop and '2-hop \& 1-hop combine' communication method. The energy balancing mechanism of this paper is based on two state thresholds and simulation result shows its superiority over the existing directional routing protocols of wireless sensor networks.
\end{abstract}

\section{KEYWORDS}

Wireless Sensor Network, Routing Protocol, Energy Efficient, Load Balancing, Directional Routing, Stationary Topology .

\section{INTRODUCTION}

Wireless sensor network (WSN) is an emerging communication technology for environmental monitoring and target tracking. It has numerous applications and placements from public to military usages and from underwater to space shuttle placements. A wireless sensor networks consists a number of sensor nodes, those are wirelessly communicated to each other and cooperatively pass data towards the base station to accomplish their dispensed responsibilities. The sensor nodes of WSN is small in size, and it consists of tiny dimension of battery for power supply, small memory chip to store data and routing table, and radio interface to send and receive signals. As the sensor nodes have limited battery power, it is not feasible or possible to recharge the batteries of sensors, such as the sensors of underwater sensor networks, battle field sensors, natural disaster prevention and monitoring sensors and implantable bio-sensors. So, energy efficient communication methods are indispensable for WSNs [2].

Routing and data disseminations are the focal causes of energy consumption of WSNs. For effective routing protocols efficient data dissemination can reduce unbalanced energy consumption of sensor nodes in a significant amount. The nodes of sensor networks can be stationary with respect to environment or can be mobile with dynamic environmental perspective [11]. As a sensor node has small memory capacity, so the large routing table driven routing procedures are not suitable for WSNs. Thus, this paper proposed an energy balanced non-table driven routing protocol for stationary WSNs.

David C. Wyld et al. (Eds) : CCSIT, SIPP, AISC, PDCTA, NLP - 2014

pp. 153-167, 2014. (C) CS \& IT-CSCP 2014

DOI : $10.5121 /$ csit.2014.4213 
Sensor nodes of a wireless sensor networks generally work as a unit of a system to complete certain obligations. Shutdown of any sensor nodes from the network creates data deficiency, and as a result the whole sensor network produces an erroneous result, incorrect and imperfect vision of environment and network is became paralyzed. In some applications, like in biomedical sensor networks, the consequence of paralyzed network cases death penalty. A routing method, which ensures balanced consumption of energy among the sensor nodes of the WSNs is essential.

\section{RELATED WORKS}

Energy efficiency is not only an issue of wireless sensor networks, it also a challenging issues in all forms of networks to meet the green communication requirements. Efficient routing protocol ensures the efficient and energy-aware communication in wireless sensor networks. Routing protocols of wireless sensor networks have been studied in reference [1] with introducing some challenges and future directions. Partial differential equation based geographical routing is proposed in reference [2]. The model is dependent on a central node, which collects the position information, residual energy information and then determines the routing path based on the proposed algorithm. The proposal is based on centralized control unit, which is not suitable for the WSNs, where there is no central node. A cluster [3][4] based and threshold sensitive routing protocol is presented in reference [5], where the authors consider power availability, nodes position, and reachability factors to determine the routing path by using cluster head. Though this proposal achieved energy efficiency but the proposal didn't concentrate on networking life time.

A hybrid routing protocol for WSNs is presented in reference [6], which allows a comprehensive information retrieval of environmental analysis and facilitate users to query of past, present and future data. This is also an application specific and cluster based routing protocol, which is focused on efficient path finding by maintaining energy-efficiency but not concerning about network life time. Some other cluster based routing protocol also proposed in reference [7] and [8]. Greedy perimeter stateless routing approach for wireless networks is proposed in reference [9], where it considers the position of source and destination to send data packets, they also presented better results than shortest-path and ad-hoc routing protocols in respect of routing protocol overhead, packet delivery rate and path length. They didn't consider energy efficiency and energy balancing issues in their routing protocols. The security gaps, and possible attacks of wireless sensor networks routing are studied in reference [10], the study presented the countermeasures and challenges of designing routing protocol with ensuring security of the data packets travelling through huge nodes of WSNs.

Power efficient topologies for sensor networks are presented in reference [11], where the authors proposed directional source aware routing protocol (DSAP) and deploy it in different 2D and 3D static network topologies to study power efficient network topology. Though the presented DSAP minimizes the energy consumption of the nodes of considered networks, but DSAP could not ensure energy balancing among the nodes of WSNs.

\section{SYSTEM MODEL}

The system model of the proposed energy efficient and load balanced routing protocol (EELBRP) for wireless sensor network is discussed in this section. Wireless sensors are deployed in various patterns based on application requirements. This paper considers that the deployment of sensor nodes follows two-dimension (2D) topology as on DSAP. The neighbour nodes are defined on the basis of 1-hop communication model and 2-hop communication model. 


\subsection{1-Hop Communication Model}

In 1-hop communication model, shown in figure 1, a sensor node has maximum eight neighbours within direct communication range. Between the node and the neighbours nodes can participate in transmissions, receptions and forwarding of data. When a node transmits packet to a neighbour node other eight neighbours can overhear the packet. In transmission, a node expenses energy for running transmitter circuits $\left(E_{T x_{-} \text {circuit }}\right)$ and also expenses energy for sending packets to distance d1 that is one hop distance amplification energy $\left(E_{T x_{-} a m p l i f i e r}\right)$ cost. Thus, to transmit $b$ bits of packet to its 1-hop neighbour, transmitter node expenses total $E_{T x_{-} \text {tcost }}$ energy by equation (1).

$$
E 1_{T x_{-} \text {trost }}(b)=E 1_{T_{\text {m_circuit }}} * b+E 1_{\text {Tx_amplifier }} * b * d_{1}^{2}
$$

As all the 1-hop neighbour nodes from the sender, overhear the $b$ bits, $n_{l}$ shows the number of neighbours except the receivers. The total overhearing energy cost of all neighbour nodes $\left(E_{R x_{-} \text {tcost }}\right)$ is equivalent to the total energy consumption of $n_{1}$ receiver circuits ( $E_{R x_{-} \text {circuit }}$ ) as formulated in equation (2).

$$
E_{\text {Ris_tegst }}(b)=n_{1} * E_{\text {lix_circuit }} * b
$$

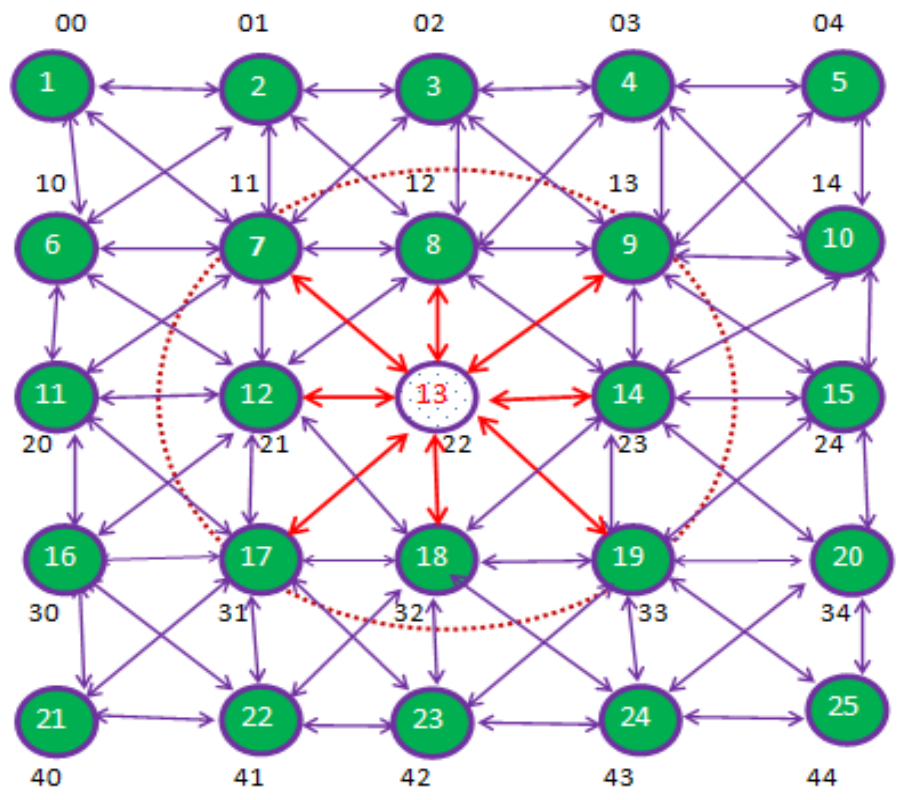

Figure 1. 1-Hop communication model for EELBRP

\subsection{2-Hop Communication Model}

The 2-hop communication model for the proposed EELBRP is shown at figure 2, where sensor node has maximum twenty neighbours. When any node transmits packet other neighbours can overhear the packet.

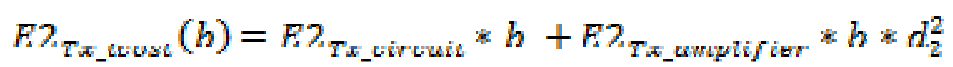

In case of transmission, a node not only expenses energy for running transmitter circuits $\left(E_{T x_{-} \text {circuit }}\right)$ but also expenses energy for sending packets to distance $d_{2}$ that is amplification energy ( $E_{T x_{-} \text {amplifier }}$ ) cost to transmit packet over $d_{2}$ distance. Thus, to transmit $b$ bits of packet to any of its 2-hop neighbours, the transmitter node expenses total $E_{T x_{-} \text {tcost }}$ energy by equation (3). 
In 2-hop communication model, all the 1-hop neighbour nodes $n_{l}$, and the nodes situated far from distance $d_{1}$, also distance $d_{2}$ nodes $n_{2}$; overhears the $b$ bits of packet, so the total overhearing energy cost of all neighbour nodes $\left(E 2_{R x_{-} t c o s t}\right)$ is equivalent to the total energy consumption of $\left(n_{1}\right.$ $+\mathrm{n}_{2}$ ) receiver circuits ( $\left.E_{R x_{-} \text {circuit }}\right)$ as formulated in equation (4).

$$
E 2_{\text {Rx wuse }}(b)=\left(n_{1}+n_{2}\right) * E_{\text {Rxx wir will }} * b
$$

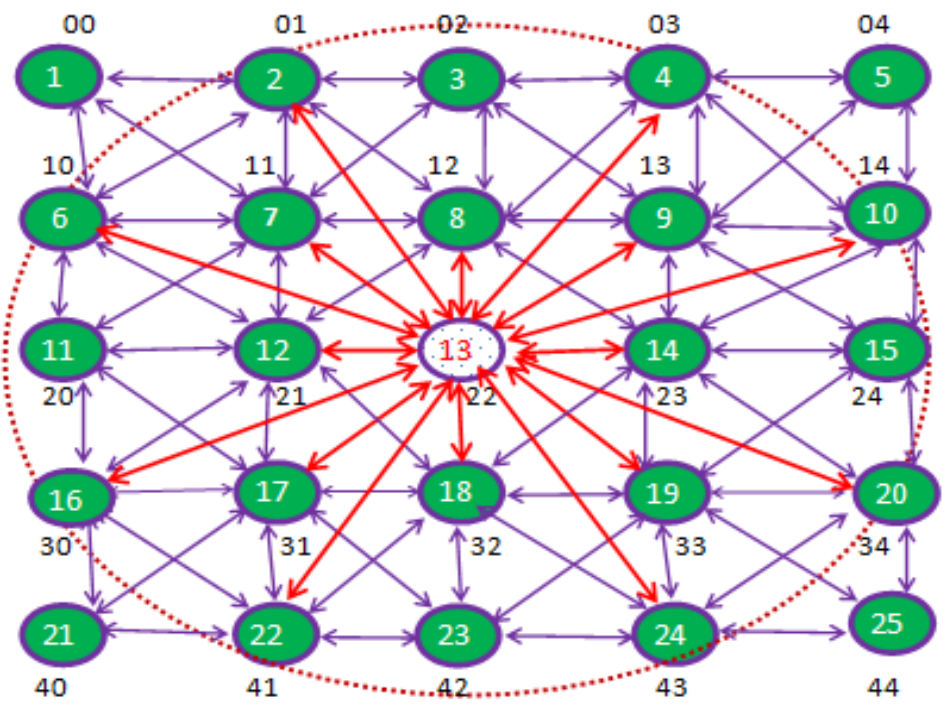

Figure 2. 2-Hop communication model for EELBRP

\section{Routing Protocol for Energy EFficiency ANd LoAd Balancing}

In this section, the detail discussion of the proposed EELBRP is presented.

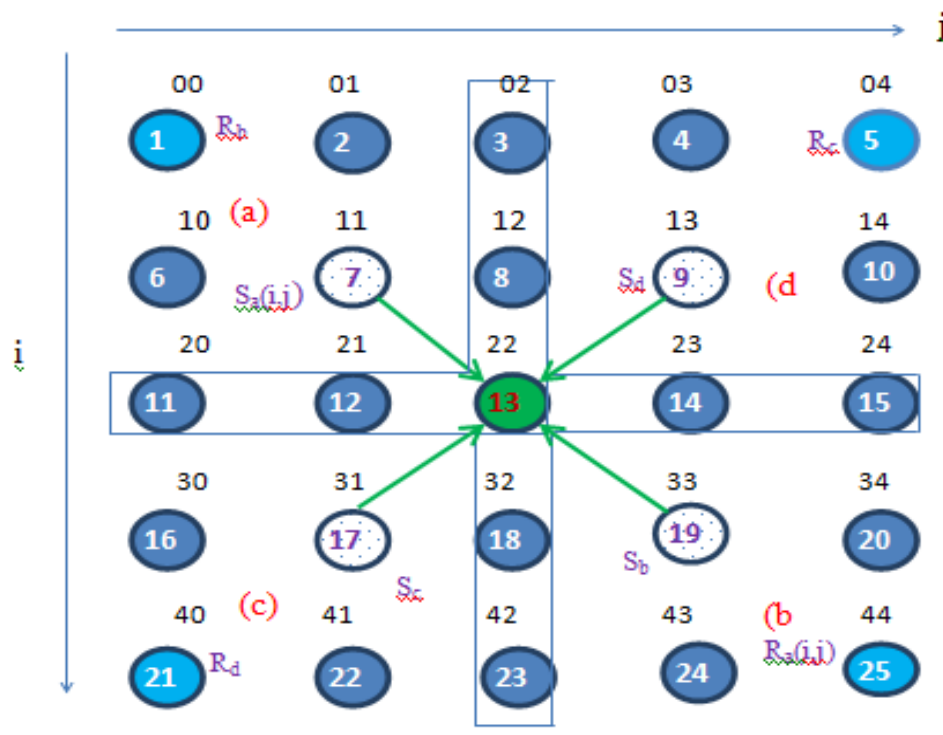

Figure 3. Direction of destination node from source node in 1-hop communication model

The proposed EELBRP is a directional routing protocol, corresponding to the direction of receiver node; it selects the forwarder nodes from a feasible set of forwarders. 


\subsection{Routing Procedure for 1-Hop communication Model}

Consider the case (a) of figure 3 , where the sender node $S_{a}$ is 7 and destination node $R_{a}$ is 25 . To find out the direction of the receiver node, the Cartesian coordinates of sender node $S_{a}(i, j)$ and the destination node $R_{a}(i, j)$ is compared according to algorithm 4.1.

In algorithm 4.1, two direction variables are D1 and D2, and L, R, D, U represent of left, right, down and up respectively. Based upon the determined values D1 and D2, the feasible set of forwarder are selected and called as the adjacent list for 1-hop communication Adi_PR1 and that is Adj_PR1_a $=\{\mathrm{D} 1 \mathrm{R} 1, \mathrm{R} 1, \mathrm{D} 1, \mathrm{U} 1 \mathrm{R} 1, \mathrm{D} 1 \mathrm{~L} 1\}=\{13,8,12,3,11\}$ is for case $(\mathrm{a})$, where $\mathrm{u} 1=\mathrm{i}-1$, $\mathrm{d} 1=\mathrm{i}+1, \mathrm{~L} 1=\mathrm{j}-1$, and $\mathrm{r} 1=\mathrm{j}+1$.

Similarly, the adjacent list of prioritized nodes for 1-hop communication of case (b), (c), (d) is defined respectively as

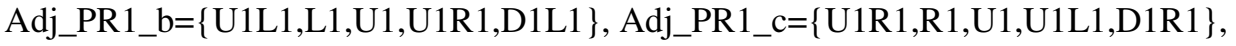
Adj_PR1_d= $\{$ D1L1,L1,D1,D1R1,U1R1 $\}$.

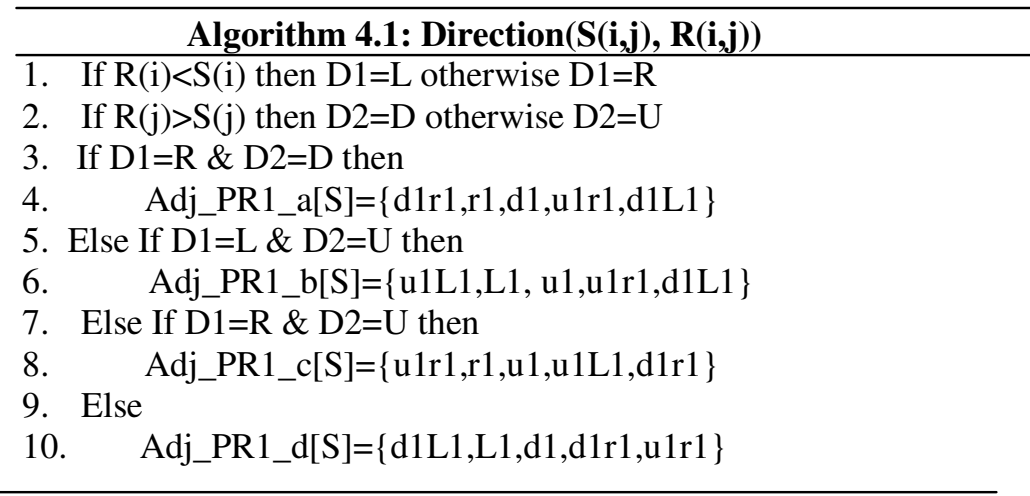

Among the feasible set of forwarder of Adj_PR1, the best suitable forwarder is selected based upon the logical distance or air distance or admissible heuristic distance from probable forwarder to the destination, which is determined in algorithm 4.2. As the assumed WSNs deployed using 2D and stationary topology, each and every node has a logical Cartesian coordinates to find out logical distance $L d$ using equation number (5)

$$
\operatorname{Ld}[v] \leftarrow \sqrt{(R(i)-v(i))^{2}+\left(R(j)-v(j)^{2}\right.}
$$

Where $R(i, j)$ is the logical coordinate of receiver node and $v(i, j)$ is the logical coordinate of feasible forwarder.

\begin{tabular}{l}
\hline Algorithm 4.2: $\operatorname{Relax}(\mathbf{R}, \mathbf{v})$ \\
\hline $\operatorname{Ld}[\mathrm{v}] \leftarrow \sqrt{\left(R(0)-v(i)^{2}+\left(R()-v(j)^{2}\right.\right.}$ \\
\hline
\end{tabular}

After finding the logical distances from list of feasible forwarder nodes to receiver nodes, the node with minimum distance is selected as the most suitable forwarder, maintain a minimum priority queue of suitable forwarders. The routing method is presented in algorithm 4.3 is an alias of Dijkstra's algorithm, where $T$ stands for topology or given WSNs , N[T] represents the nodes of the topology $T$, and variable Route gradually stores the routing path from sender to receiver. 


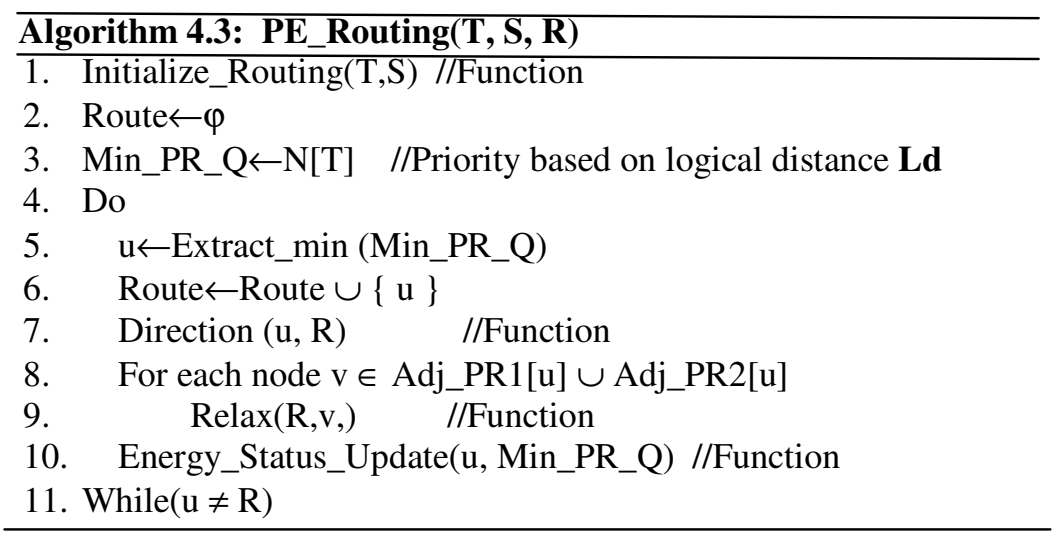

Algorithm 4.3, consists a procedure for initialization of nodes residual energy variable Res_energy as maximum energy of the batteries of sensor nodes at starting time. Logical distances from sender to all other nodes are also initialized as infinite at the beginning of routing procedure in algorithm 4.4 .

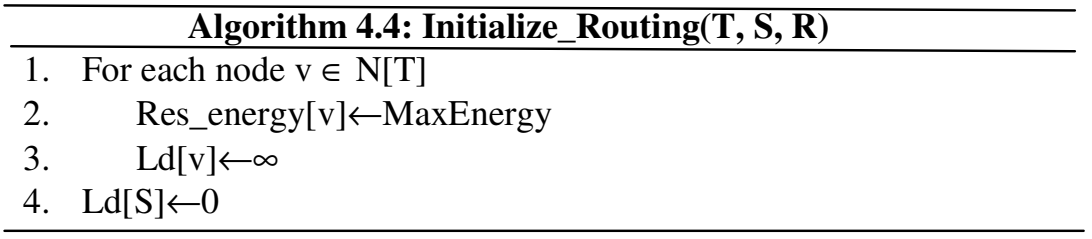

Algorithm 4.3, also consists an energy status updating function for dynamic updates of sensor nodes residual energy after sending and receiving of data packets. For changing the energy status of receiver (forwarder) nodes, all the 1-hop neighbor nodes (Adj1) are considered because of the broadcasting nature of WSNs. Algorithm 4.5 is designed with the energy model formulated in equation (1) and (2).

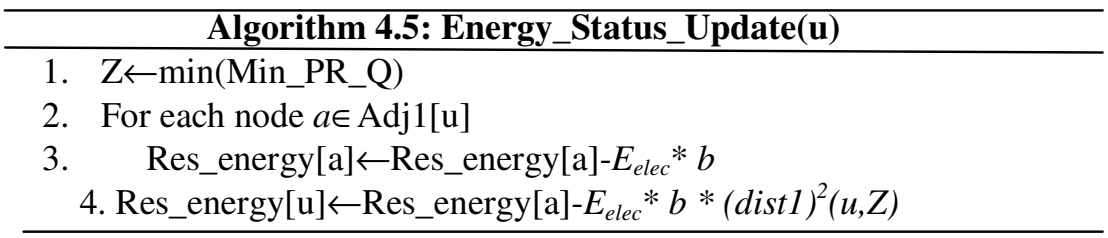

\subsection{Routing Procedure for 2-Hop\& 1-hop Combine Communication Model}

The routing procedure for 2-hop \& 1-hop combine communication model of proposed EELBRP is little bit different from 1-hop communication model. In this combine communication model sender node always tries to send packets to its one hop neighbours first for forwarding. The direction of destination node will need to be determined here also in combine model. The procedure of determining the direction of receiver node is defined in algorithm 4.6.

Consider the case (a) of figure 4, where sender node $S_{a}$ is 7 and receiver node $R_{a}$ is 25 , we follow almost same procedure to determine the direction as we discussed in section 4.1, but the feasible set of forwarders are also included not only the 1-hop but also the 2-hop nodes, which we call the adjacent list of prioritized nodes for 2-hop communication Adi_PR2 and that is

Adj_PR2_a $=\{d 2 \mathrm{r} 1, \mathrm{r} 2 \mathrm{~d} 1, \mathrm{~d} 2, \mathrm{r} 2\}=\{18,14,17,9\}$, where $\mathrm{d} 2=\mathrm{i}+2, \mathrm{r} 2=\mathrm{j}+2, \mathrm{u} 2=\mathrm{i}-2$, and $\mathrm{L} 2=\mathrm{j}-2$. Similarly, the adjacent list of prioritized nodes for 2-hop communication of case (b), (c), (d) is 


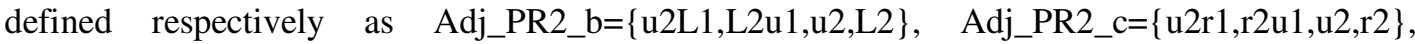
Adj_PR2_d $=\{$ d2L1,L2d1,d2,L2 $\}$.

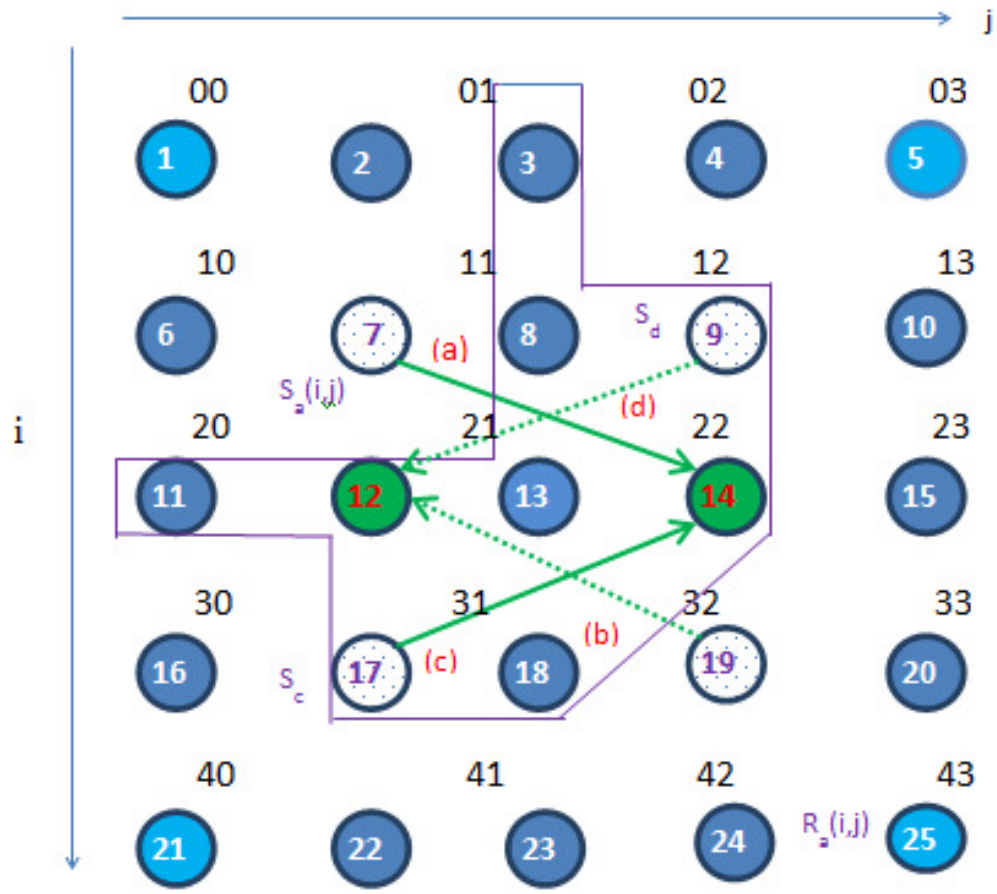

Figure 4. Direction of destination node corresponding to source node in 2-hope \& 1-hop combine communication model

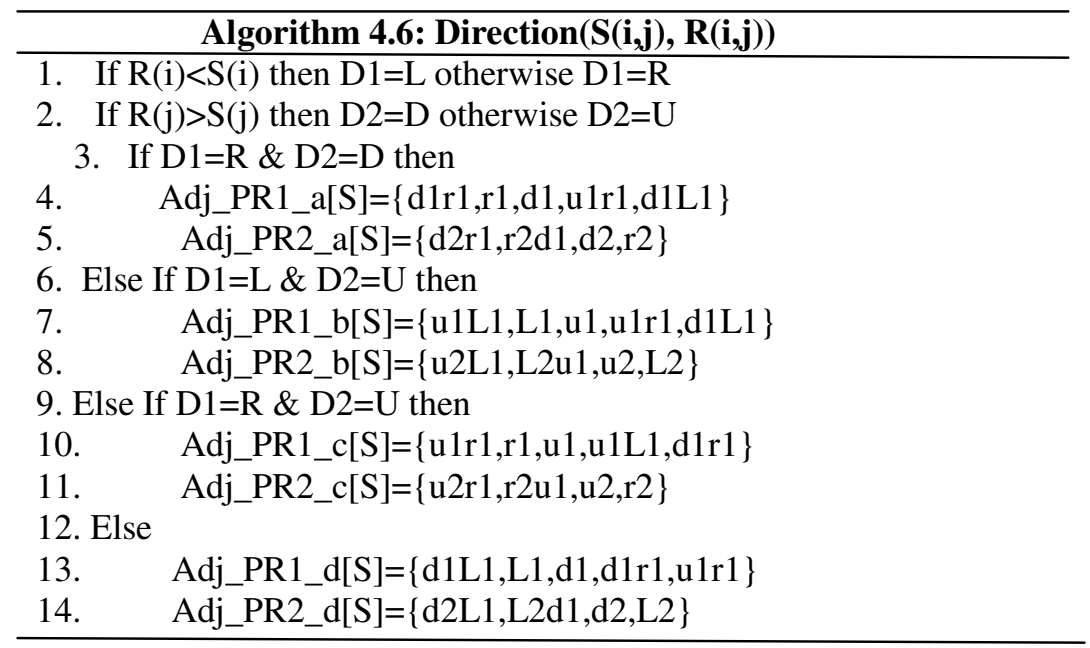

Among the feasible set of forwarders of Adj_PR1 and Adj_PR2, the best suitable forwarder is selected based upon the logical distance from probable forwarder to destination, which is determined in algorithm 4.7, the logical distance of destination node from 1-hop feasible forwarders will get higher priority as their distance is customized with negative sign. 


\begin{tabular}{|c|}
\hline Algorithm 4.7: Relax(R, v) \\
\hline 1. IF $v \in$ Adj_PR2[u] \\
\hline $\mathrm{Ld}[\mathrm{v}] \leftarrow\left(\sqrt{ }(R(i)-v(i))^{2}+(R(j)-v(j))^{2}\right)$ \\
\hline 3. Else \\
\hline $\mathrm{Ld}[\mathrm{v}] \leftarrow-\sqrt{ }(R(i)-v(i))^{2}+(R(j)-v(j))^{2}$ \\
\hline
\end{tabular}

Among the determined logical distances, the feasible forwarder node with minimum distance is selected as the suitable forwarder towards the receiver. Algorithm 4.8 presents the suitable forwarder selection procedure considering both of the feasible set of forwarders of Adj_PR1 and Adj_PR2 at line number 8 .

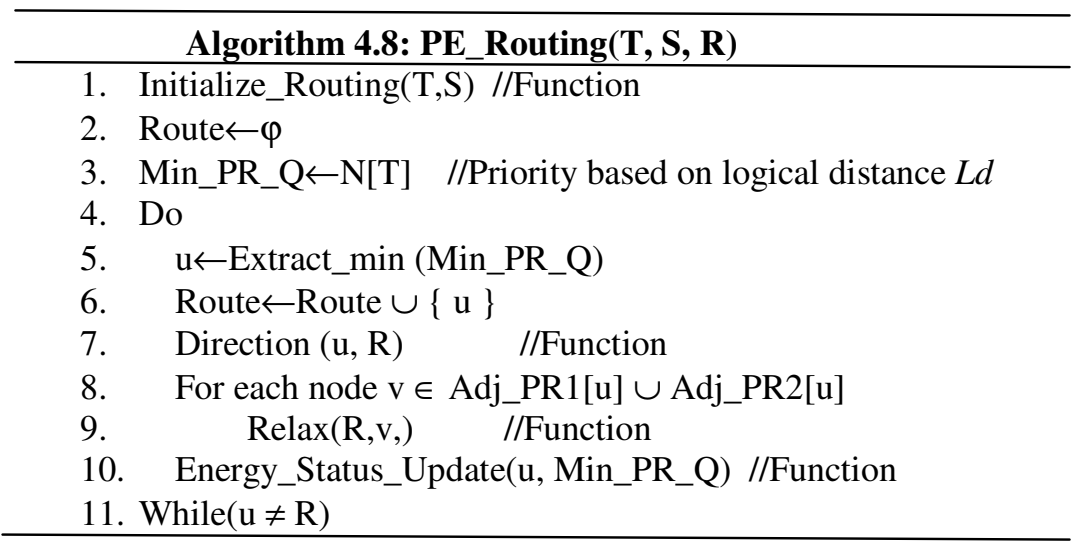

Algorithm 4.8 also calls the energy status updating procedure, which is presented in algorithm 4.9. For changing the energy status of receiver (forwarder) nodes, all the 1-hop neighbor nodes (Adj1) and 2-hop neighbor nodes (Adj2) are considered because of the broadcasting nature of WSNs. Algorithm 4.9 is designed with the energy model formulated in equation (3) and (4).

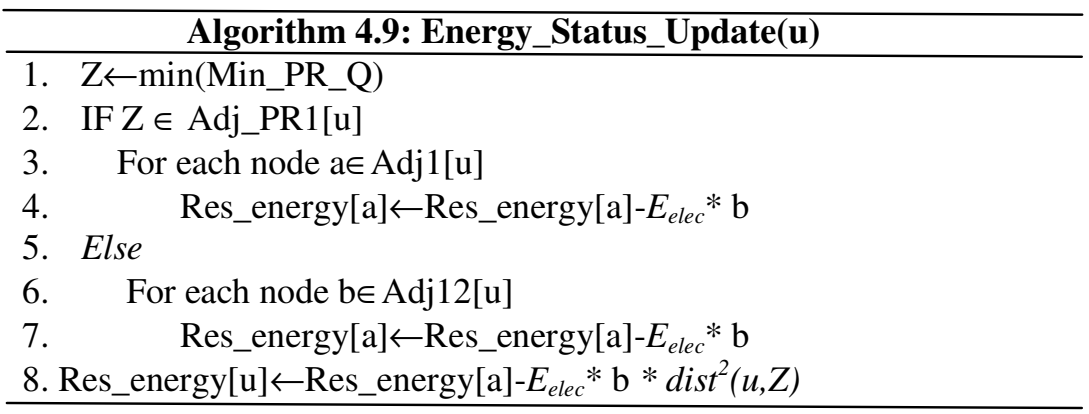

\subsection{Routing Procedure for 2-Hop\& 1-Hop Combine Communication Model with Energy-Awareness}

To design an energy aware and energy balanced routing protocol the 2-hop \& 1-hop combine communication model of figure 4 is used. As the Relax function is the controlling function of suitable forwarder node selection, the Relax function is designed accordingly in algorithm 4.10 to select forwarders in energy-efficient manner. If residual energy of the prioritized neighboring node of sender is greater than the threshold-1 (th1) then just follow 2-hop \& 1-hop combine routing procedure to select forwarders, but if residual energy of all of the prioritized neighboring node of sender is less than the threshold-1 (th1) but greater than threshold-2 (th2) then determine energy ratio of each of the node, and update logical distance variable based on energy ratio. So, in such case the routing algorithm 4.8 tries to balance energies of the whole network between 
threshold-2. Finally, if the residual energy of the entire prioritized neighboring node of sender is less than the threshold-2 then algorithm determines, which node consumes less energy i.e which node has much residual energy and update logical distance accordingly. By this procedure, the node with highest residual energy among feasible forwarder list Adj_PR1 and Adj_PR2 placed in the 1st position of the minimum priority queue as well as selected as the best suitable forwarder for balancing energies among its neighbors.

\begin{tabular}{|c|c|}
\hline \multicolumn{2}{|c|}{ Algorithm 4.10: Relax(R, v) } \\
\hline 1. IF Res_e & energy $[v]>$ th1 \\
\hline IF ve & $E$ Adj_PR2[u] \\
\hline 3. & $\mathrm{Ld}[\mathrm{v}] \leftarrow-\left(\sqrt{(R(x)-v(i))^{2}+(R(y)-v(j))^{2}}\right)$ \\
\hline Else & \\
\hline 5. & $\mathrm{Ld}[\mathrm{v}] \leftarrow \sqrt{(R(x)-v(i))^{2}+\left(R(y)-v(0)^{2}\right.}$ \\
\hline 6. Else IF & th $2<$ Res_energy $[\mathrm{v}]<$ th 1 \\
\hline 7. & 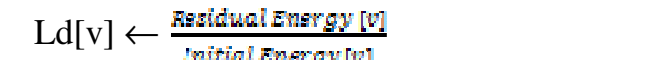 \\
\hline 8. Else & \\
\hline 9. & Ld[v] $\leftarrow$ Initial_Energy[v]-Res_Energy[v] \\
\hline
\end{tabular}

\section{SIMULATION RESULTS}

To evaluate the performance of the proposed EELBRP algorithm, the MATLAB R2010a simulation tools are used with $\mathrm{C}++$ simulation program to analyze the energy efficiency and balancing status. The simulation scenario is presented in Table 1 .

Table 1. Simulation scenario for performance study of EELBRP algorithm

\begin{tabular}{|l|l|l|}
\hline Simulation Parameters & Symbols & Values \\
\hline Topology & $2 D$ & $\begin{array}{l}\text { 8 neighbors within } 1 \text { hop } \\
20 \text { neighbors within } 2 \text { hop }\end{array}$ \\
\hline Number of nodes & $n \times n$ & $11 \times 11$ \\
\hline $\begin{array}{l}\text { Horizontal (or vertical) } \\
\text { distance between two nodes }\end{array}$ & $d_{1}$ & 0.5 meters \\
\hline Packet size & $b$ & 512 bits \\
\hline Total number of packets & $P$ & 10,000 \\
\hline Transmitter circuitry energy & $E_{T x_{-} \text {circuit }}$ & $50 \mathrm{~nJ} / \mathrm{bit}$ \\
\hline Transmitter amplification energy & $E_{T x_{-} \text {amplifier }}$ & $100 \mathrm{pJ} / \mathrm{bit} / \mathrm{m}^{2}$ \\
\hline Receiver circuitry energy & $E_{R x_{\_} \text {circuit }}$ & $50 \mathrm{~nJ} / \mathrm{bit}$ \\
\hline Threshold-1 & th1 & $53 \mathrm{~J}$ \\
\hline Threshold-2 & th2 & $25 \mathrm{~J}$ \\
\hline
\end{tabular}

The performance of proposed EELBRP is studied using two essential performance metric of wireless sensor networks i.e energy efficiency and network lifetime. Figure 5 and figure 6 shows the performance of the EELBRP algorithm without using energy balancing procedure. Between these two figures, the figure 5 shows the residual energies of each of the $11 \mathrm{X} 11=121$ nodes of the wireless sensor networks after handling 10,000 packets of 512 bits and following 1 hop communication method. The total energy consumption is 4228.360266 Joules and residual energy is balancing between 27 to 95 Joules. 
Whereas, figure 6 shows the residual energies of each nodes of the wireless sensor networks after handling same number of packets of 512 bits and following 2 hop communication method. The total energy consumption is 5082.707723 Joules and residual energy is balancing between

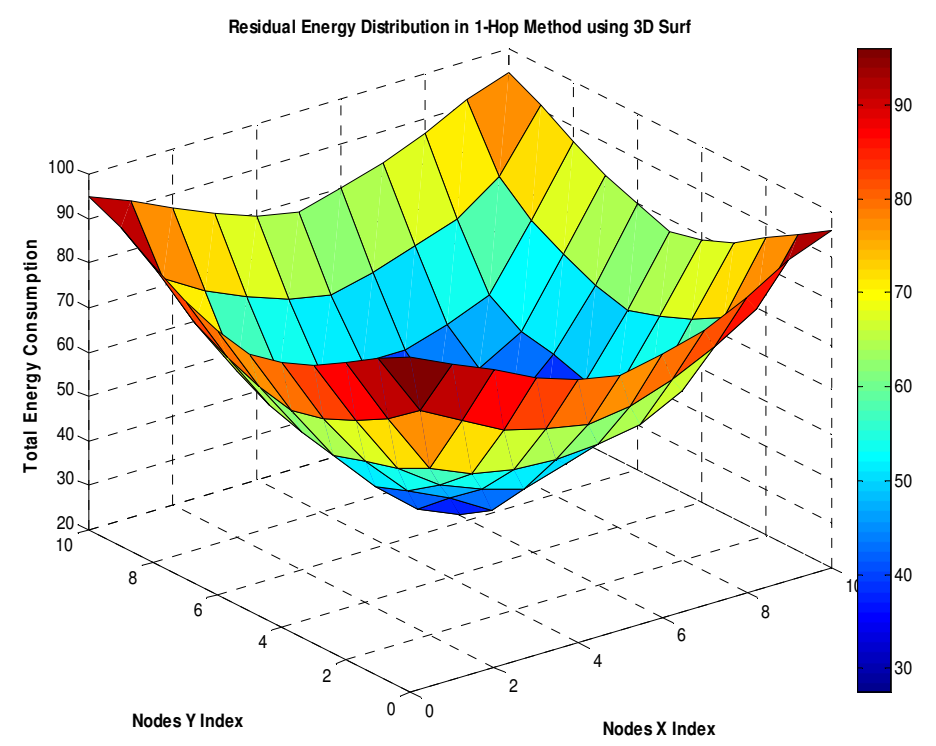

Figure5. Residual Energy of each nodes using EELBRP (1-Hop case)

21 to 90 Joules. In comparison between 1-hop and 2-hop communication method, following the EELBRP without energy balancing procedure, 1-hop communication method consumes less energy than 2-hop communication method. In comparison of energy balancing performance, in both 1-hop and 2-hop cases the energy difference remains around 70 Joules, which means some of the network nodes dies very firstly then other nodes, and network become paralyzed with short period of time i.e performance of EELBRP without energy balancing procedure is not impressive in respect to network lifetime.

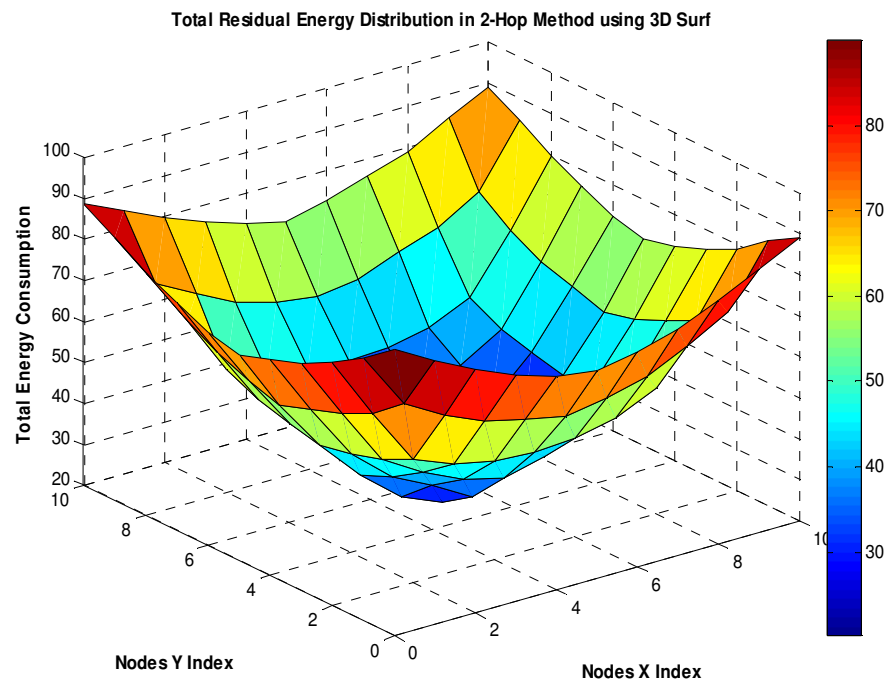

Figure 6. Residual Energy of each nodes using EELBRP (2-Hop Case) 
Figure 7 shows the residual energy distribution the nodes of the wireless sensor networks following the proposed EELBRP with energy balancing procedure, where 1-hop and 2-hop combine communication model is used rationally to improve network lifetime. The total energy consumption is 4660.138730 Joules and residual energy is balancing between 65 to 92 Joules. In comparison to 1-hop communication method, it consumes little bit more energy but in comparison to 2-hop communication method, it consumes less energy.

But in comparison of energy balancing performance, the residual energy difference among the sensor nodes of the network is 27 Joules, which means the EELBRP with energy balancing procedure enhances network lifetime significantly with sacrificing limited total network energy.

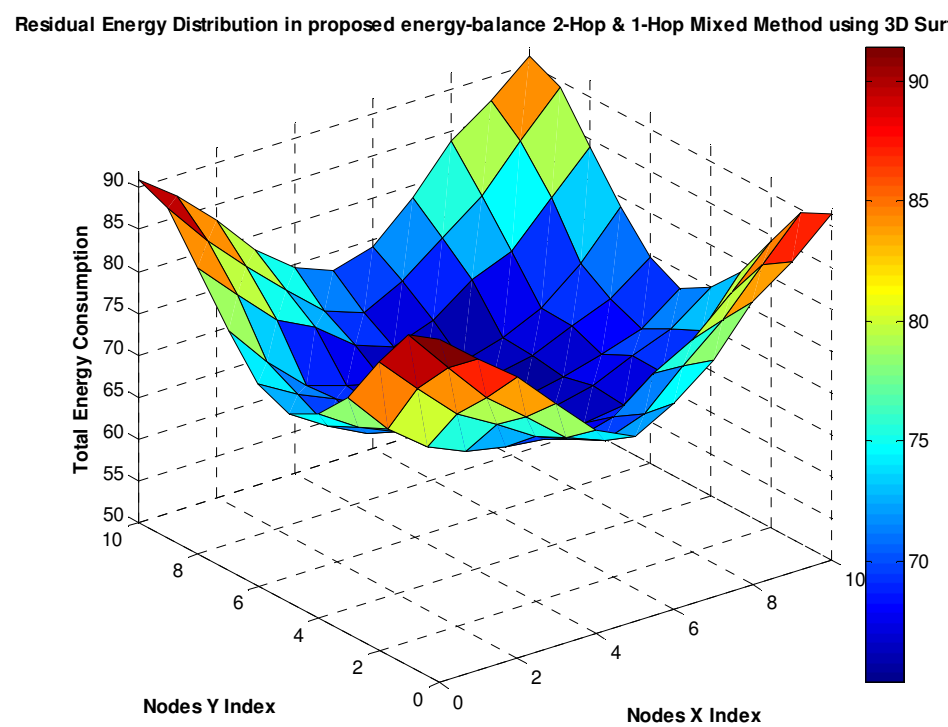

Figure 7. Residual Energy of each nodes using EELBRP (2-Hop \& 1-Hop combine with energy balancing procedure, where threshold $1=53$ Joules and threshold $2=25$ Joules)

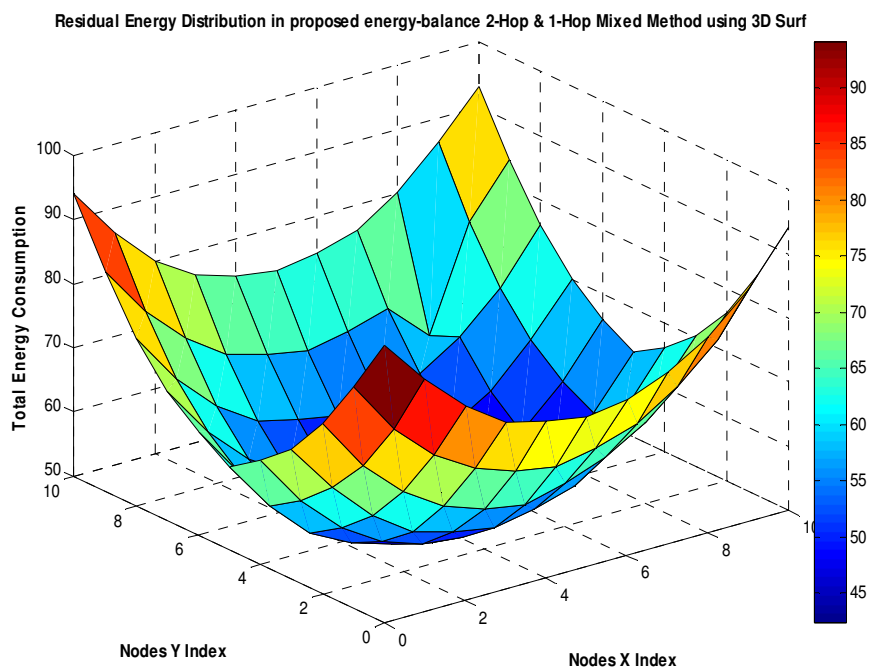

Figure 8. Residual Energy of each nodes using EELBRP (2-Hop \& 1-Hop combine with energy balancing procedure, where threshold $1=40$ Joules and threshold $2=5$ Joules) 
The performance of the EELBRP with energy balancing procedure is also evaluated using different threshold values. Figure 7 9 shows the network energy performance graph of various threshold conditions, where the threshold 1 with 53 joules and threshold 2 with 25 joules clearly shows their suitability and justification of threshold selection in figure 7.

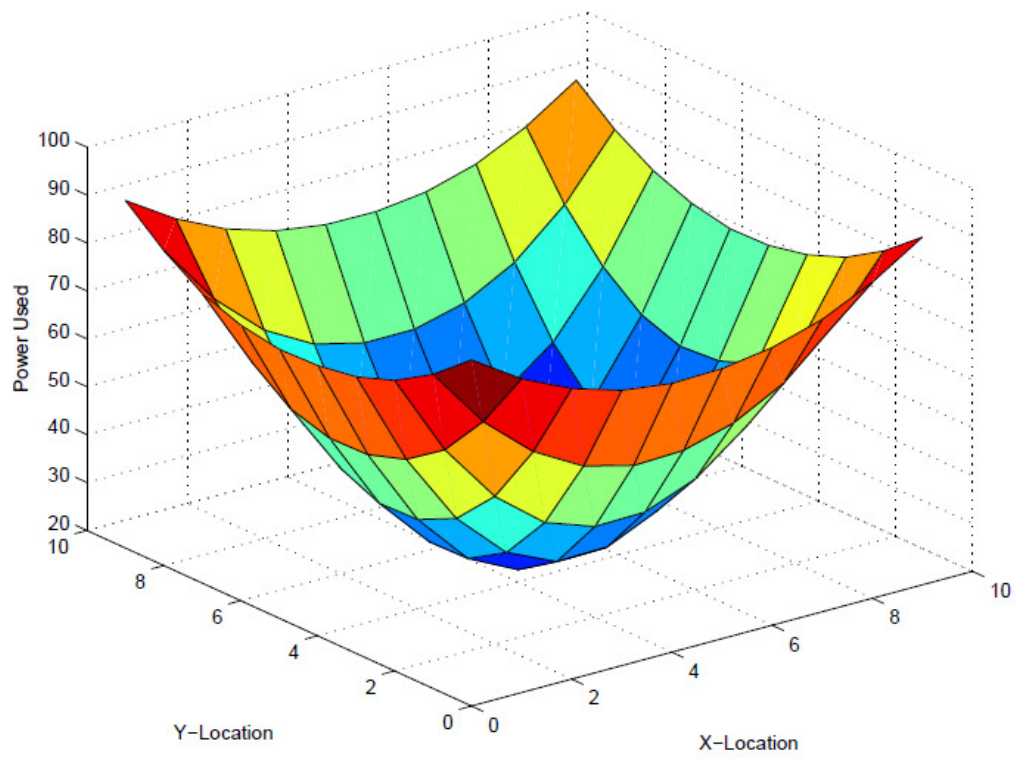

Figure 9. Residual Energy of each nodes using EELBRP (2-Hop \& 1-Hop combine with energy balancing procedure, where threshold $1=60$ Joules and threshold $2=35$ Joules)

Table 2. Comparison of Total Energy Consumption among 2-hop, 1-hop \& "2-hop + 1-hop combine with energy balancing" method

\begin{tabular}{|c|c|c|c|c|}
\hline $\begin{array}{l}\text { Threshold } 1 \text { and } \\
\text { threshold } 2\end{array}$ & $\begin{array}{c}\text { Energy } \\
\text { Consumption }\end{array}$ & $\begin{array}{c}\text { 2-hop } \\
\text { communication } \\
\text { Method }\end{array}$ & $\begin{array}{c}\text { 1-hop } \\
\text { communication } \\
\text { method }\end{array}$ & $\begin{array}{l}\text { Proposed } \\
\text { EELBRP } \\
\text { with energy } \\
\text { balancing }\end{array}$ \\
\hline \multirow[t]{2}{*}{$40 \mathrm{~J}$ and $5 \mathrm{~J}$} & $\begin{array}{c}\text { Total } \\
\text { Consumption }\end{array}$ & $5082.70772 \mathrm{~J}$ & $4228.36026 \mathrm{~J}$ & $\begin{array}{r}4357.4121 \mathrm{~J} \\
\text { Less } \\
\text { consumption } \\
\end{array}$ \\
\hline & $\begin{array}{l}\text { Residual } \\
\text { Energy } \\
\text { distribution }\end{array}$ & $21 \sim 90$ & $27 \sim 95$ & $\begin{array}{r}42 \sim 94 \\
\text { Imbalance } \\
\text { energy } \\
\end{array}$ \\
\hline \multirow[t]{2}{*}{$53 \mathrm{~J}$ and $25 \mathrm{~J}$} & $\begin{array}{c}\text { Total } \\
\text { Consumption }\end{array}$ & $5082.70772 \mathrm{~J}$ & $4228.36026 \mathrm{~J}$ & $\begin{array}{r}4660.1387 \mathrm{~J} \\
\text { Moderate } \\
\text { consumption } \\
\end{array}$ \\
\hline & $\begin{array}{l}\text { Residual } \\
\text { Energy } \\
\text { distribution }\end{array}$ & $21 \sim 90$ & $27 \sim 95$ & $\begin{array}{r}65 \sim 92 \\
\text { Balanced } \\
\text { energy } \\
\end{array}$ \\
\hline \multirow[t]{2}{*}{$60 \mathrm{~J}$ and $35 \mathrm{~J}$} & $\begin{array}{c}\text { Total } \\
\text { Consumption }\end{array}$ & $5082.70772 \mathrm{~J}$ & $4228.36026 \mathrm{~J}$ & $\begin{array}{l}\text { 4983.0121J } \\
\text { Higher } \\
\text { consumption } \\
\end{array}$ \\
\hline & $\begin{array}{l}\text { Residual } \\
\text { Energy } \\
\text { distribution }\end{array}$ & $21 \sim 90$ & 27 95 & $\begin{array}{l}\text { 69 94 } \\
\quad \text { Balanced } \\
\text { energy }\end{array}$ \\
\hline
\end{tabular}

The performance study of EELBRP algorithm is summarized in table 2, where the proposed EELBRP with energy balancing strategy shows its energy balancing power with moderate energy 
consumption. The 2-hop and 1-hop communication method using EELBRP without energy balancing procedure shows similar energy consumption disregarding threshold values because EELBRP without energy balancing procedure has no option of selecting energy threshold values.

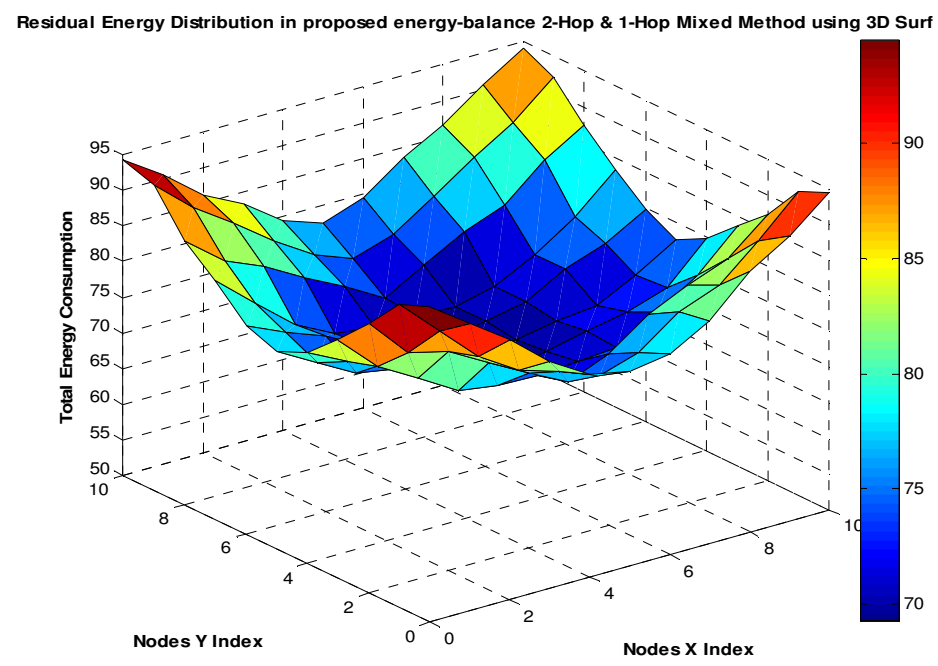

Figure 10. Residual energy of each node using DSAP method

The performance of proposed EELBRP algorithm is also compared with the existing benchmarked DSAP routing algorithm, because DSAP also directional and concerned about stationary network topologies like EELBRP. Following 1-hop communication and using same simulation scenario of EELBRP simulation study, the residual energy graph is presented in figure 10. The figure shows that DSAP balancing residual energy distribution between 20 to 89 Joules and the total energy consumption is 4612.215061 Joules, whereas EELBRP balanced energy between 27 to 95 and consumes 4228.360266 joules of energy shown in figure 5 .

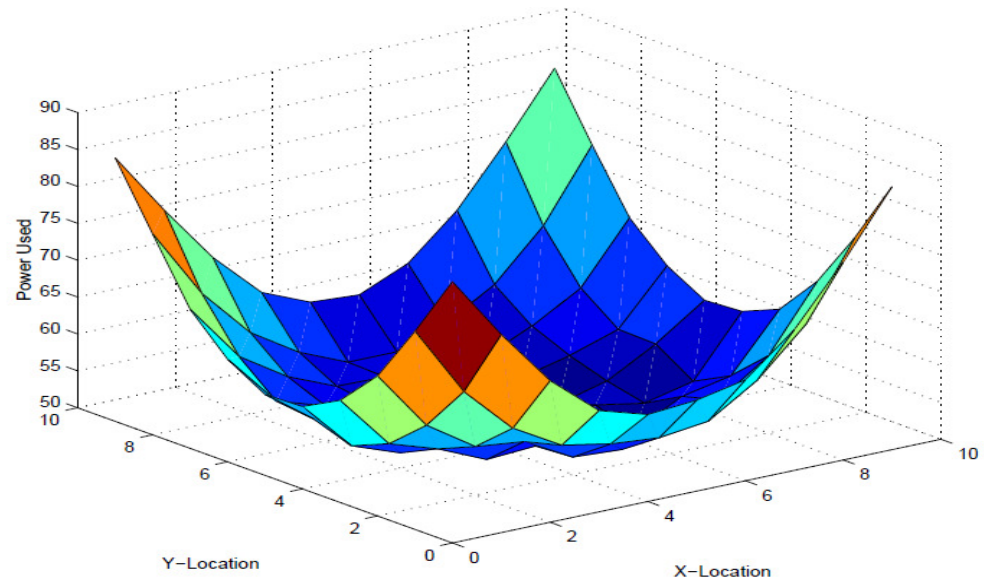

Figure 11. Residual energy graph of power-aware DSAP method

The main cause behind the lower performance of DSAP is the weakness in determination of directional values (DV) i.e considering networks connection similar like wired networks rather than using the broadcasting nature of wireless networks effectively. Conversely, EELBRP 
determine the logical distance from destination node to feasible forwarder nodes and select the best forwarder to forward data packets towards the destination node.

The performance of power-aware DSAP is also presented in figure 11. The residual energy distribution the nodes of the wireless sensor networks following power-aware DSAP is 50 to 83 and total energy consumption is 4978.735009 Joules. On the other hand, the proposed EELBRP with energy balancing procedure keeps lower bound of residual energy to 65 and upper bound to 92, so energy is more balanced in EELBRP and it enhances the network lifetime as well while maintaining less energy consumption 4660.138730 Joules than power-aware DSAP.

\section{Conclusions}

Development of energy aware and energy balanced routing protocol for stationary wireless sensor networks is the major significant contribution of this study. Considering the ability of dynamic energy changing capability of sensor nodes, the presented routing protocol is simulated in 1-hop, 2-hop and "2-hop \& 1-hop combine" communication method. The proposed EELBRP shows improved performance by accepting and combining with energy balancing and energy efficiency perspectives. As the proposed routing protocol is directional, the use of directional antenna surely reduces the energy consumption of the network in a significant rate.

\section{ACKNOWLEDGEMENTS}

This work has been funded by the BK21+ program of the National Research Foundation (NRF) of Korea.

\section{REFERENCES}

[1] Al-Karaki, Jamal N., and Ahmed E. Kamal. "Routing techniques in wireless sensor networks: a survey." Wireless Communications, IEEE 11.6 (2004): 6-28.

[2] Kalantari, Mehdi, and Mark Shayman. "Energy efficient routing in wireless sensor networks." Proc. of Conference on Information Sciences and Systems. 2004.

[3] Mary Wu, InTaek Leem, Jason J. Jung, and ChongGun Kim. "A resource reuse method in cluster sensor networks in ad hoc networks." In Intelligent Information and Database Systems, pp. 40-50. Springer Berlin Heidelberg, 2012.

[4] Mary Wu, Byung Chul Ahn Kim, and Chong Gun,. "A channel reuse procedure in clustering sensor networks." Applied Mechanics and Materials 284, pp: 1981-1985, 2013.

[5] Manjeshwar, Arati, and Dharma P. Agrawal. "TEEN: A Routing Protocol for Enhanced Efficiency in Wireless Sensor Networks." IPDPS. Vol. 1. 2001.

[6] Manjeshwar, Arati, and Dharma P. Agrawal. "APTEEN: A Hybrid Protocol for Efficient Routing and Comprehensive Information Retrieval in Wireless Sensor Networks." ipdps. Vol. 2. 2002.

[7] Heinzelman, Wendi Rabiner, Anantha Chandrakasan, and Hari Balakrishnan. "Energy-efficient communication protocol for wireless microsensor networks."System Sciences, 2000. Proceedings of the 33rd Annual Hawaii International Conference on. IEEE, 2000.

[8] Heinzelman, Wendi B., Anantha P. Chandrakasan, and Hari Balakrishnan. "An application-specific protocol architecture for wireless microsensor networks." Wireless Communications, IEEE Transactions on 1.4 (2002): 660-670.

[9] Karp, Brad, and Hsiang-Tsung Kung. "GPSR: Greedy perimeter stateless routing for wireless networks." Proceedings of the 6th annual international conference on Mobile computing and networking. ACM, 2000.

[10] Karlof, Chris, and David Wagner. "Secure routing in wireless sensor networks: Attacks and countermeasures." Ad hoc networks 1.2 (2003): 293-315.

[11] Salhieh, Ayad, et al. "Power efficient topologies for wireless sensor networks. "Parallel Processing, International Conference on, 2001.. IEEE, 2001. 


\section{AUTHORS}

\section{*Corresponding Author:}

ChongGun Kim received the B.E and the M.E degree in Electronic Engineering from YeungNam University, Korea in 1981 and 1987 respectively. He received $\mathrm{Ph} . \mathrm{D}$ degree in Computer Science and Information Mathematics from University of ElectroCommunications, Tokyo, Japan, in 1991. He has been serving as a Professor at the Department of Computer Engineering, Yeungnam University since March 1991. In his doctoral work, he was engaged in research on load balancing of distributed computing systems. He was a visiting scholar of Virginia Tech., USA and UCSC, USA in 1996 and 2003. His current research interests include Computer Networks, Wireless Sensor Networks, Network Security, and Distributed Computing Systems.

Alghanmi Ali Omar received the B.S. degree in Computer Science from Kyung Hee University, Korea in 2012. He received King Abdullah Scholarship from Riyadh, Saudi Arabia for his Bachelor studies in Computer Science. Currently, he is pursuing his master degree in Computer Engineering at Yeungnam University in Korea. He also received King Abdullah Scholarship for his Master studies in Computer Engineering. His areas of research interests include Wireless Sensor Networks, Network Security, Information Security, and Energy Balancing Mechanism.
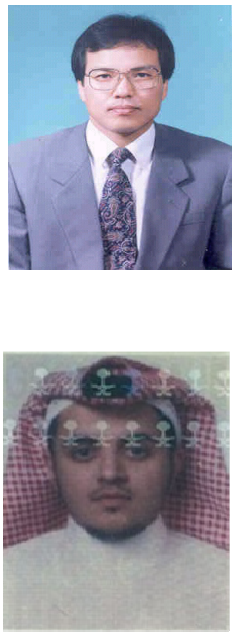